\title{
Reflections on Multidisciplinary Teamwork: From Experience to Impact
}

\author{
Krista Liguori, Molly Eckman, Khanjan Mehta \\ Humanitarian Engineering \& Social Entrepreneurship (HESE) Program \\ The Pennsylvania State University \\ University Park, PA 16802 \\ Correspondence: khanjan@engr.psu.edu
}

\begin{abstract}
The Humanitarian Engineering and Social Entrepreneurship (HESE) Program at Penn State engages students and faculty across the university in rigorous research, design, field-testing, and launch of technology-based social enterprises. The complex nature of the real-world problems tackled by HESE ventures requires engagement from students and faculty across the university. Such interdisciplinary teamwork is fraught with issues stemming from differing academic traditions, vocabularies, communication, work habits, and most importantly, philosophies of entrepreneurial engagement in developing countries. This reflection article, based on interviews with 22 highly engaged students and recent alumni, provides a candid reflection on the structures and practices within the HESE program that facilitate or, at times, impede multidisciplinary teamwork. The methods and approaches described in this article are not best practices. Rather, they are simply practices and lessons learned that have emerged over time, and continue to evolve, as students and faculty in HESE march forward on their learning, research and entrepreneurial engagement continuum.
\end{abstract}

Index Terms: Engaging Students, Social Entrepreneurship, Multidisciplinary Teamwork, Eteams

\section{INTRODUCTION}

Several academic programs and co-curricular student clubs have emerged to address the needs of developing communities in the United States and across the world. The Humanitarian Engineering and Social Entrepreneurship (HESE) program at Penn State engages students and faculty across the university in rigorous research, design, field-testing, and launch of technologybased social enterprises. These enterprises address challenges in resource-constrained settings, mostly in sub-Saharan Africa. HESE ventures are multi-year endeavors that range from low-cost greenhouses and solar food dryers to telemedicine systems, ruggedized biomedical devices, cellphone applications, and informal education systems. HESE espouses the fundamental philosophies of Empathy, Equity and Ecosystems to create a vibrant and emergent framework for students, faculty, and diverse partners to come together and collaborate to develop sustainable and scalable solutions. Building long-term relationships with multi-sectoral partners and leveraging indigenous knowledge to foster developmental entrepreneurship form the foundation of all initiatives. 
While the program practices the pedagogy of Service-Learning to further the social ventures, the word "service" is rarely used. The focus of the program is not to "serve" anyone but to build equitable reciprocal relationships with diverse partners. Students and faculty work shoulder-toshoulder with partners to develop technologies and launch entrepreneurial ventures that prioritize the social returns while being economically sustainable. Sustainability, in this context, refers to the notion that solutions must be technologically appropriate, socially acceptable, environmentally benign, and economically sustainable. Rather than striving to solve each partner's specific problem(s), the preferred approach is to work with a larger set of partners to launch independent enterprises that are replicable and scalable. For example, instead of building an affordable greenhouse for a community partner, venture teams work with local startups, industry partners, agricultural research institutes, government ministries, non-profits and other entities to build independent enterprises that manufacture, sell, and support greenhouses for the larger market. In essence, the goal is to co-create products and services with diverse stakeholders and commercialize them to benefit larger populations.

The majority of HESE ventures over the last five years have focused on global health and food security challenges. The complex nature of these real-world problems requires expertise and engagement from students and faculty across the university. The HESE program has been successful in this convergence of disciplines, cultures, countries, and epistemologies. Typically, half of the approximately 80 students engaged in the core teams every year are engineering students, with the rest of the them representing every other college across campus (Figure 1). Over the last seven years, more than half of the students have consistently been women. The program attracts a significant number of honors scholars as well as high-performing students. Over the past decade, HESE ventures have collectively touched the lives of a few million people across Kenya, Tanzania, Rwanda, India, U.S., Jamaica, Cameroon, and other countries. Some ventures have failed while others are on the slow but steady path towards sustainable existence and scaling up to become multi-million smile enterprises. In addition, HESE students are concurrently engaged in original publishable research in a wide swath of topics, ranging from innovative technologies, entrepreneurship pedagogies, systems thinking, telemedicine, cellphones, social networks and trust, indigenous knowledge systems and development, innovation in engineering education, and food value chains. The resulting peer-reviewed publications inform and inspire other social innovators. Since 2009, over 80 peer-reviewed articles have been published in journals and conference proceedings, most of them with students as lead authors. 


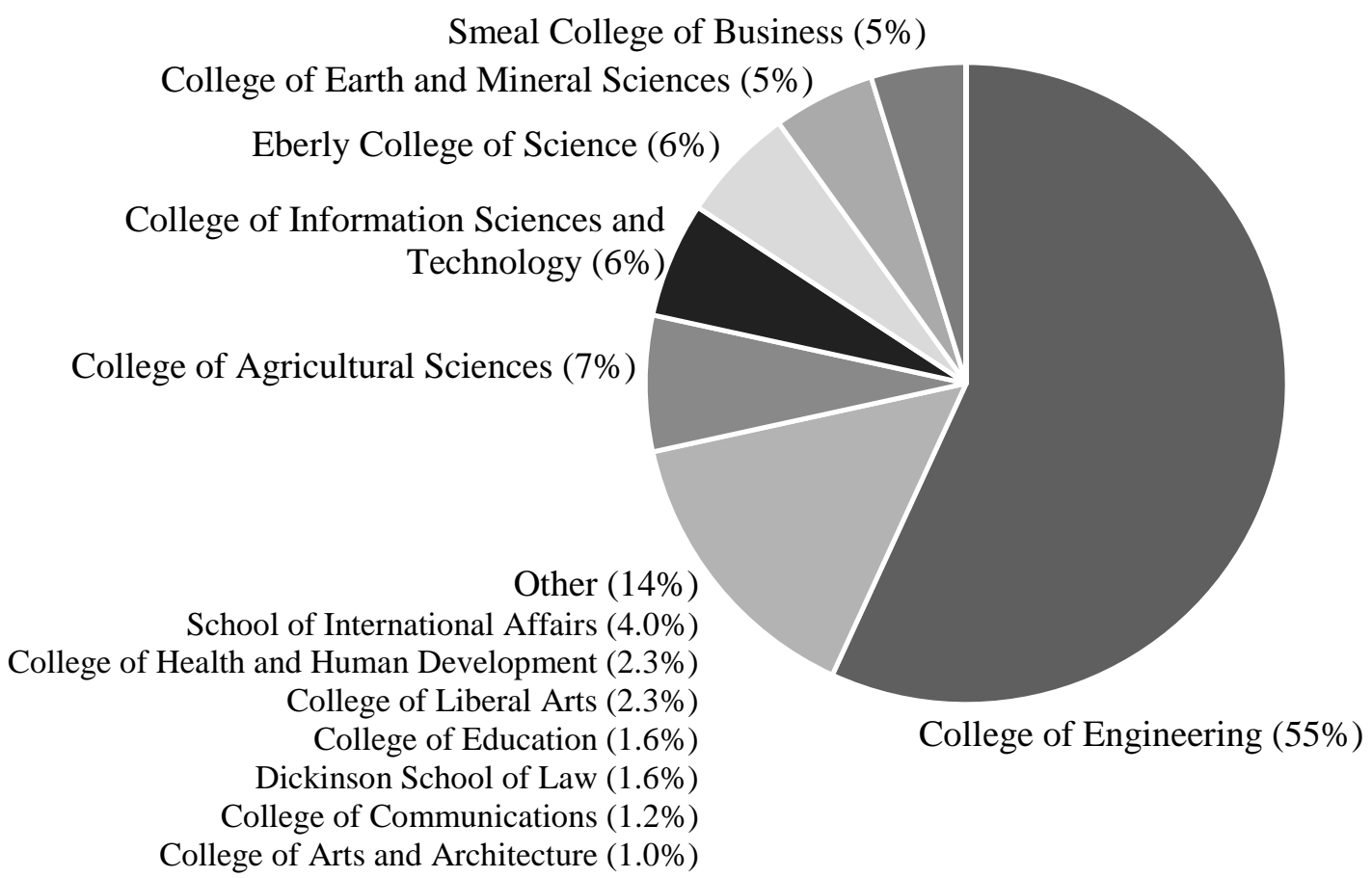

FIGURE 1

ACADEMIC REPRESENTATION IN THE HESE PROGRAM

This type of radical collaboration is essential to advance ventures forward and has been pivotal in entrepreneurial advances and research accomplishments. At the same time, such interdisciplinary teamwork is fraught with tensions stemming from differing academic traditions, vocabularies, communication and work habits, and most importantly, differing philosophies of engagement with community partners. This reflection article, based on interviews with 22 highly engaged students and recent alumni, provides a candid reflection on the structures and practices within the HESE program that facilitate or, at times, impede multidisciplinary teamwork. Off the interviewees, $68 \%$ were actively engaged in at least one project at the time of interview, and $41 \%$ were female. This is not a scientific study and no approval was sought from the Institutional Review Board (IRB) for human subjects research at Penn State. The approaches presented in this article are not any sort of best practices either. These are simply practices and lessons learned that have emerged over time, and continue to evolve, as students and faculty in HESE march forward on their learning, research and entrepreneurial engagement continuum.

\section{Organization OF THE HESE Program}

\section{Course structure}

HESE classes are experiential learning forums with an open environment nurtured by mutual respect between the instructors and students. HESE ventures are completely embedded in a series of five courses (Figure 2). Students who opt to take all five courses receive a certificate in 
Engineering and Community Engagement. They also have the option of combining these courses with other foundational courses to receive a minor in Entrepreneurship and Innovation with a specialization in Social Entrepreneurship. HESE course offerings include Social Entrepreneurship [ENGR 451: 3 credits], Projects in Humanitarian Engineering [EDSGN 452: 2 credits], Design for Developing Communities [EDSGN 453: 1 credit], HESE Fieldwork Experience [EDSGN 454: 0.5 credit], and HESE Reflection and Research Dissemination [ENGR 455: 3 credits]. During May and June, students travel to partnering communities, typically for a period of three weeks, to advance their ventures. Every year, 1-3 students who have successfully completed HESE coursework are recruited as Fellows. After graduation, HESE Fellows spend up to one year (typically $4-6$ months) working on-site with partners to advance ventures forward and coordinate with design teams at the university. The programmatic learning outcomes for students encompass Human-Centered Design, Context-Driven Design, Social Entrepreneurship, Global Engagement, Systems Thinking, Ethical Reflection, Multidisciplinary Teamwork, Communication, and Scholarly Research and Publication. Students engage in the HESE program in different ways, such as honors theses, HESE courses, and embedded projects in non-HESE courses. While approximately 80 students are in the core HESE teams at a given time, crosscurricular integration academically engages over 500 students every year.
Fall Sem.
Spring Sem.
Maymester
Fall Sem.

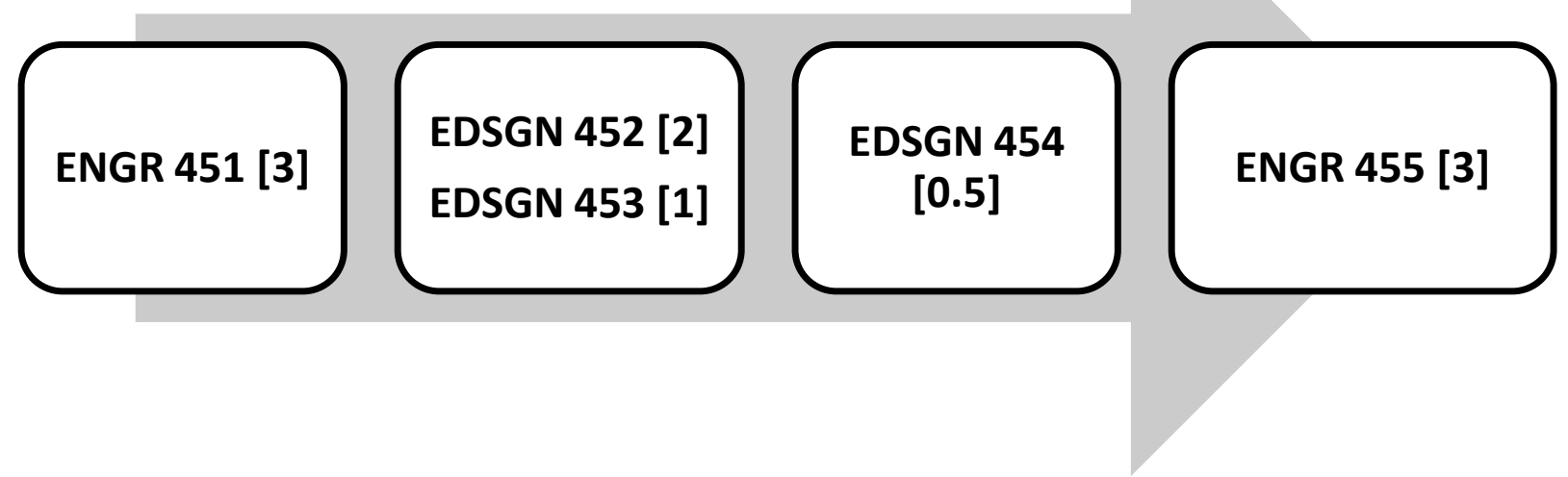

FIGURE 2

RECOMMENDED SEQUENCE OF HESE COURSES

Students can enter the HESE program in the Fall semester through the ENGR 451 course or in the Spring through concurrent enrollment in the EDSGN 452 and EDSGN 453 courses. These courses are open to undergraduate and graduate students from all disciplines. The only prerequisite is fifth semester standing and lower division students with international community engagement experience are routinely admitted. The ENGR 451 class is focused on developing business and implementation strategies for technology-based social ventures while the EDSGN 452 course emphasizes product development and preparation for fieldwork. In the Spring semester, the core teams are housed in the EDSGN 452 class with students concurrently enrolled in the one-credit EDSGN 453 seminar class. The weekly seminar is streamed live (and is available on-demand) for students at other campuses, counterparts abroad, and industry professionals mentoring the teams. Besides the core teams housed in the EDSGN 452 classes, a 
number of other courses participate in the various ventures. These courses include the Introduction to Biomedical Engineering Research and Design (BME 401) class, few sections of the freshman-level Introduction to Engineering Design (EDSGN 100) class, Technical Writing (ENGL 202C) class, etc. Students who have successfully completed the EDSGN 452 and 453 courses can then conduct fieldwork on faculty-led trips housed in the EDSGN 454 course. Only students who have completed the fieldwork course are permitted into the ENGR 455 course that focuses on ethical decision-making, grassroots diplomacy and research dissemination as it applies to the praxis of social entrepreneurship. HESE emphasizes evidence-based approaches and leverages research publications as the baton from one team to another. The ENGR 455 course provides students a series of workshops and faculty mentoring to develop the scholarly manuscripts that are then turned in to conferences and journals for review.

HESE is a professional program designed to prepare students for careers as social entrepreneurs or in the broader social innovation and international development arena. As part of their professional preparation, students participate in university-wide competitions such as 1,000 Pitches, Ag Springboard, and Undergraduate Research Expo as well as departmental competitions like the Mechanical and Nuclear Engineering (MNE) Innovation Competition. Outside the university, students travel to conferences such as the IEEE Global Humanitarian Technology Conference, Venture Well (formerly National Collegiate Inventors and Innovators Alliance (NCIIA)) Annual Meeting, and the Humanitarian Technology Conference. Students routinely participate in competitions like the American Society of Mechanical Engineers (ASME) I-Show, Biomedical Engineering (BME) Idea Competition, Alleviating Poverty Through Entrepreneurship (APTE) Competition, Global Idea to Product Competition, and the Agricultural Innovation Showcase. Conferences and competitions bring together experts and practitioners in the humanitarian engineering and social entrepreneurship domains, allowing students to exchange ideas and experiences while developing their professional networks. This real-world calibration is especially empowering to students as it provides validation of their work and builds up their confidence in pursuing a career in international development. Such professional engagement expands student and faculty horizons and keeps the program constantly growing and evolving.

\section{Team structure}

Core teams for each venture emerge through an organic process that takes into account project needs and student interest. Ongoing ventures and ideas for potential new projects are presented during the first few weeks of the semester, and students are given the opportunity to sign up for a project of interest. If students are not drawn to a particular project, they are assigned to teams based on the unique needs of each project. This process maintains continuity while ensuring a new cohort of students is incorporated into each venture. The average early-stage team has 3-5 students, and teams tend to grow as ventures move through their lifecycles, with mature ventures often having upwards of ten students to satisfy the larger workload. HESE teams are largely based on the concept of E-teams (multidisciplinary group of students, faculty, and mentors working together to bring an invention to market) championed by Venture Well.

Venture teams 'own' and direct relevant work every semester and then pass the baton on to the next team. HESE ventures are validated and developed further by teams, until they succeed, fail, or are deemed not worth pursuing further by faculty and experienced students. The decision on continuation or suspension of a venture is driven by validation and success within the market 
and the ability to garner necessary funding and intellectual resources. While there are several early-stage ventures, the real-world organically funnels and advances fewer ventures on to more advanced phases. Current ventures in the design or scale-up phase include inkjet-printed test strips for screening people for Urinary Tract Infections (UTIs) and diabetes, low-cost 3D printed prosthetics, using hand grip strength as a health screening tool, telemedicine systems, affordable greenhouses, mango waste briquetting machines, and an electronic payments system for smallholder farmers.

Ventures are funded primarily by a series of external grants. For example, the affordable greenhouse venture was validated by an internal seed grant of \$1,000. An EPA P3 grant $(\$ 15,000)$ facilitated technology development while an NCIIA Sustainable Vision grant $(\$ 40,000)$ enabled rigorous field-testing and commercialization in Kenya, Tanzania, Rwanda and Cameroon. Finally, a Securing Water for Food (SWFF) grant $(\$ 500,000$ over three years) from USAID awarded in collaboration with a non-profit organization, World Hope International, is helping establish for-profit greenhouse enterprises in Mozambique and Sierra Leone. Alongside, a private foundation is supporting greenhouse pilots in Egypt while another foundation is bankrolling a pilot program in India. The grant proposals as well as concept notes (for Foundation funding) were developed by HESE teams in collaboration with relevant partners.

\section{Venture Fieldwork}

A subset of students who have participated in the EDSGN 452 and 453 courses travel to the project site for three weeks at the end of the spring semester to advance their ventures. Students are required to sign up for the 0.5-credit HESE Fieldwork Experience (EDSGN 454) course. Each year, approximately 40 students choose to travel. The majority of the HESE ventures are based in Kenya with ongoing operations in Sierra Leone, Mozambique, Cameroon, Tanzania, Rwanda, and India. Funding for the trip, which costs approximately $\$ 3,500$, is the students' responsibility. Most students take advantage of travel grants available through their respective departments, colleges, research institutes as well as external funding sources. The HESE program provides modest funding support $(\$ 300$ - \$600) to students who intend to stay engaged over multiple semesters. While most students tend to get at least one-third of their travel funding covered, a few diligent and persistent students get their entire travel expenses covered. The research component of the ventures opens up additional funding sources. As the program has become more well-known within the university and global programs have been prioritized, more departments are willing to fund student travel at higher levels.

While working in-country, students expand their teams by integrating members from relevant partnering organizations. Faculty, Fellows and experienced students serve as liaisons with venture partners and help the teams bridge communication and expectation gaps. Research teams have access to translators if necessary. These expanded cross-functional teams have a multipronged daily agenda that covers their ventures and research projects. All the teams come together for a meeting every evening to debrief from the day's activities, discuss challenges and recalibrate action plans. The field experience is also a rich environment for students to explore the ethical intricacies of engaging in projects in international contexts. Students engage in debates on ethical issues related to science, technology and society in an applied setting - the people are real, the ethical question is real and most importantly, a consensus is required to decide on the further course of action. These three weeks in-country tend to be extremely busy as teams go into overdrive to achieve venture outcomes and collect data for the research endeavors. 


\section{REFLECTION ON TEAMWORK SUCCESSES ANd CHALlENGES}

This article is a reflection of the past ten years of the HESE program as seen through the perspective of the students and the program coordinator. For this article, 22 HESE students and alumni were interviewed about their engagement in this learning community. These students reflected on their experiences and were encouraged to analyze why conflicts occurred and how they could improve their teamwork. The alumni compared their experiences in HESE during their undergraduate years with graduate school experiences and other entrepreneurial / industry experiences. These narratives were then synthesized and categorized into observations related to 1) academic structure, 2) roles and responsibilities, 3) building a culture, 4) working in teams. This reflection incorporates successful strategies to promote multidisciplinary teamwork while also acknowledging areas for improvement. As discussed earlier, this article is not being presented as a source of best practices. Rather, the goal is to share unique experiences in creating social impact with highly interdisciplinary teams of students, faculty, ground partners, and external advisers working together towards a common goal.

\section{Academic Structure}

HESE teams require a diverse range of knowledge, skills and abilities to accomplish necessary tasks. Engaging motivated people with different areas of expertise and a diversity of interests on the team helps speed along specialized tasks, such as having a bioengineering major working in the lab to design the necessary chemical reactions for a test-strip indicator. A team may include a liberal arts student who handles the entrepreneurial aspects of the project while an engineering major may already have the connections and expertise to work in a faculty member's lab to develop a novel device. However, in many cases, students work in areas drastically different than their official majors. A common occurrence is engineering and science majors who develop an interest in entrepreneurship and spend their energies developing and validating business models. This type of transdisciplinary work, in which the unique interests and skills of each student are utilized regardless of major, benefits both the students by giving them diverse experience and the progression of the venture by using a play-by-strength method to optimize results.

The HESE program is led by one professor who teaches the courses, coordinates the ventures, and facilitates the fieldwork. This simplifies the hierarchical structure by providing students one main point of contact for evaluating proposals, making resource decisions and handling conflicts and discrepancies. This clear structure helps enormously when conducting fieldwork and making time-sensitive or complex entrepreneurial decisions. The model is analogous to having a single CEO in a company or an odd number of members on the board of directors. While teams make decisions by consensus most of the time, there are times when a clear vote or veto is needed. Another reason for each team having a lead mentor is that most ventures are funded by grants awarded under the supervision of a faculty Principal Investigator (PI). Individual teams are granted a significant amount of autonomy and have full responsibility for the success or failure of their projects. As long as the team is making progress and communicating it effectively, lead mentors step back from day-to-day activities and focus on more strategic and systemic issues.

Other faculty members and external professionals routinely take on duties as collaborators, advisors, and referees. At the same time, team-teaching of core courses is avoided altogether as it leads to confusion and tension in a fast-paced entrepreneurial setting. Rather students have 
access to a pool of subject-matter experts and the decision on the path forward rests with the team and the lead mentor. Advisers lend their expertise in a particular subject to answer a specific question the team has. As experts in their own respective fields, academic technical advisers might lack an entrepreneurial perspective and entrepreneurs might not have a good understanding of how things work in developing countries. Students are advised to become adept at gleaning the information they need from various advisors without getting bogged down in conflicting opinions. This is particularly important because the majority of the advisers developed their expertise in the U.S. context while the ventures are completely focused on developing countries. For example, medical professionals in the US are trained to make clinical decisions without considering financial implications. While physicians from the College of Medicine advise HESE teams on clinical issues, their perspectives on health-seeking behaviors and optimal courses of treatment in a specific African country are not as helpful. In this case, the perspective of local doctors and community health workers is more relevant and practical.

Conflicts often emerge when other faculty or collaborators are added to a venture for only the fieldwork experience in Africa. Last-minute additions of faculty members who have not been engaged in the venture earlier, are not experienced in working in developing countries, or are not comfortable with non-hierarchical entrepreneurial teams, generally lead to disaster. To mitigate such tensions at the most critical time in the venture lifecycle, all fieldwork participants are expected to be deeply involved in the venture during the preceding semester. This mandates everyone to have a deeper understanding of the design factors and rationale for past decisions and strategies as well as the expectations and action plan for fieldwork. More importantly, this semester-long engagement facilitates trusting relationships amongst team members and prepares them to play by strengths.

Defining the roles of partners and stakeholders within a group is also essential. This applies not only to the team members, but also faculty members and industry partners who contribute to the effort. Several faculty members and subject matter experts are engaged in HESE ventures, but they take on the roles of either a collaborator or an adviser. A collaborator in a venture provides meaningful insights, stays up-to-date on the team's progress, and has a voice in the decision-making process. They spend some time working with the team members by contributing their knowledge, experiences, or relevant research. Common sources of collaborators are faculty, graduates students, and HESE Fellows. Highly engaged students are chosen as HESE Fellows after graduation and spend up to one year working on HESE ventures abroad. They are funded by venture-specific grants or local startup partners. These Fellows are a valuable source of information due to their long-term involvement and extensive experience and fieldwork. Current and past Fellows constantly provide feedback and often contribute to ventures as advisers or collaborators.

\section{Roles and responsibilities}

The lead faculty member of the HESE program plays the roles of professor, mentor, and supervisor. He gives directions, provides useful insights into the context of the field, gives critical feedback, and contributes to the design process at different junctures. Since the grading system for the course depends largely on the input of external judges, the professor can maintain a more neutral position which allows him to integrate better with each team. In many instances, he is the only reliable judge of venture feasibility, as he is the one with the deepest understanding of the capabilities and limitations of venture partners. His primary role is to push students and 
ventures forward while keeping them solidly grounded on what is doable in the field with the resources at hand. This is done through a balance between giving broad advice and specific instructions. If a team consistently has a strong strategy and reliably produces high quality deliverables, the professor can give less direction and allow the team to progress independently. While vague guidelines and constantly changing directions sometimes frustrate students, by the end of the course they are much more comfortable with chaos and ambiguity.

The professor also encourages this lesson by playing devil's advocate and adding turbulence to proceedings, forcing students to move beyond their comfort zones and consider new directions for their projects. A team working on developing business models for nutritious pizza, for example, was prompted to address how their venture is affected by the increasing number of women in the workforce and how the venture navigates gender roles and expectations. These questions challenged the team, that was focused primarily on validating their business model and determining the nutritional content of their product. In the end, it enabled them to see their venture from an entirely different perspective and opened new pathways for economic sustainability through female street food vendors.

Students who are new to the program are generally comfortable with setting short-term goals that appear feasible within a certain time period. They don't have the experience to reasonably assess the capabilities and limitations of their teams at the university, and the larger venture teams on-site in Africa. Most new students tend to aim very low because they just don't know what is possible and have lower self-efficacy. Hence semester-end and longer-term goals are set by the lead faculty mentors in concert with HESE Fellows and experienced students familiar with current challenges as well as the successes and failures of previous ventures in a specific location. Faculty and experienced students also take the lead in identifying the next set of research questions that warrant further investigation and help new research teams develop their research plans and navigate human-subjects approvals.

The ideal framework of a sustainable venture created through the HESE program utilizes a new set of students each year with a few students rolling over from one year to the next for better continuity. Past experience dictates that it takes 3-5 years for a single venture to be successfully implemented and reach maturity in terms of impact and economic sustainability. As the only constant team member and PI on grant proposals, the lead mentor is responsible for driving the "big picture" strategy and mentoring both new and returning students to achieve it. The strategy for a venture is not only determined by what the team wants to accomplish, but what can be realistically accomplished in the target country in the allotted timeframe. Frequent conversations with field partners and Fellows validate the strategy and specific tactics. It is the responsibility of the professor and returning students to ensure that the ventures are aligned with the larger strategy. Students who stay involved for multiple semesters also serve as mentors to their newer teammates.

\section{Building a Culture}

The culture of traditional academic courses has encouraged many undergraduate students to achieve course requirements with only the minimum amount of work. Students develop the skills to complete assignments on time by directly answering prompts via regurgitation rather than engagement and deeper learning. This "skill" hinders projects in real world ventures. For this reason, the lead faculty member is responsible for setting "stretch" requirements and expectations for students. For example, teams often set the goal of completing a thorough review 
of relevant literature. While this may be one of the most challenging tasks they have ever undertaken, upon completion the professor will push them further by requiring them to synthesize that review into a publishable manuscript. Throughout the semester, strict deadlines for deliverables, which include extensive public speaking engagements, push the students to produce significant results in a short period of time. Under this pressure, many students excel, while some do not. Most student reflections expressed that their experience in HESE was "more intense" than other courses and teamwork experiences. The large majority of students that thrive under these conditions and produce consistently successful outcomes support this pedagogical method. Those students who do not excel under these rigorous conditions may choose not to enroll in additional HESE courses, and are free to leave the learning community after any one of the courses. The students who successfully complete the final HESE Reflection and Research Dissemination class (ENGR 455) have successfully navigated the prior coursework and find themselves capable of creating publishable manuscripts based on their research.

Throughout student interviews, when asked what motivated them to spend so much time on their ventures, many students replied that the professor "is scary." Students further explained that the high-expectations pushed them to achieve more because they were fully aware of the professors' as well as venture partners' expectations. Another common response was that they wanted to impress the professor or other stakeholders. The students demonstrated that they have an awareness of how much work they needed to devote in order to meet the expectations.

HESE develops a culture of due diligence by requiring students to know all details concerning their ventures. Students must be proactive and independently seek out information, often turning to outside experts and sources to fill knowledge gaps. For example, a team assessing the feasibility of using handgrip strength as a health screening tool read over one hundred scholarly articles on handgrip strength over a one month period to determine its clinical relevance. Another team in the same class spent significant amounts of time in the lab of a collaborating professor testing, iterating, and validating inkjet-printed test strips used to screen people for UTIs. A third team took extra classes in circuits and programming in order to design and build a functional data collection prototype. This high-expectations environment encourages students to build a mindset of persistence and motivation. "You do it to do it well, not to meet the deadline," explains one student. With passion and persistence, students strive by themselves, and create partnerships, to do whatever it takes and keep pivoting until they solve a problem at hand.

Throughout the HESE course pipeline, students present their venture for 5-10 minutes, followed by 30 minutes of Q\&A by a 8-12 member referee panel. These referees are experts in their field and force the students to answer the difficult questions about their venture. Each team prepares not only for their presentation but also for any other kinds of questions they may get. In the Design for Developing Communities course, students get feedback from the referee panel on how to improve their product design. Throughout the Social Entrepreneurship course, students present their social business and demonstrate potential for financial sustainability, scalability, a thoroughly-developed concept of operations, and a plan for reaching their target market. Success during these presentations requires students to back up every claim and plan with specific assumptions and evidence -- which can only be found through diligent research. During preparation for design review presentations, for example, a team developing a new type of street food, researched the necessary food preservation methods, developed recipes, created business and financial plans, and examined economic trends that affect the prices of their recipes' ingredients. They conducted sensory evaluation tests on their food product, designed the food 
carts, and created back-up slides with every minor detail related to their venture. At every juncture, students are challenged to validate and provide supporting evidence for every assumption and strategy.

A culture of frugality is fostered by placing specific price constraints on the students and their resources. For example, a team of students were challenged with designing and building health data collection devices under a Bill of Material (BOM) cost of $\$ 10$ or affordable greenhouses that could be assembled by two people in two days with materials costing less than \$200. Constraints drive creativity. Price constraints force the students to think outside the box, consider diverse design approaches and materials, and continuously strive to hit their target price. In country, the students are expected to seek the best prices and bargain with vendors in order to be frugal with the limited funding. For example, while prototyping a low-cost solar food dryer in Kenya, the team needed to find the right battery within their budget. They spent a couple of hours going shop-to-shop looking for the battery and inquiring about the price. After negotiating with multiple vendors, they successfully purchased the battery at a price slightly lower than what the locals typically pay. This survey also familiarized them with the marketplace, the kinds of products on sale, and the rampant price variations. More importantly, they reported being much more confident in navigating the marketplace, interacting with regular people, and buying materials at the right prices. Students developed their own little network in the market and countered the perception that they were amateurs who would happily pay the quoted price. These kinds of experiences are critical to understanding "how things work" in different cultural contexts and developing low-cost products that meet the people's needs.

Programs like HESE are unique in that students are able to work on ventures that impact real people. Students clearly see the potential impact of their ventures, which increases their motivation and leads to larger time and effort investments in their ventures. One student described his HESE experience as, "the most meaningful, applicable, down-to-earth work I've done in a while." Many other students describe the program as "life-changing." Traveling to the partnering communities and working with the people broadens students' understandings of "impact". Another student noted that he was more committed to his venture after traveling because only then was he able to fully grasp the purpose of the program. "Instead of doing work for a grade, we're doing work to make a difference in someone's life," explains a HESE alumna. The real-world applications of the HESE program promote professionalism among the students and discourage unconstructive behaviors, such as social loafing or constant tension within a team. Students reflected that in this learning community, "you can't slack off anymore, you have to pull through on your commitments. Everyone has to do their part here or it won't work." Lessons in ethical decision making and grassroots diplomacy are integrated into the curriculum to increase student understanding of the decision-making process in the field. In contrast to traditional academic courses focused on student learning, the primary focus of the HESE program is on creating sustainable impact. This is prioritized over personal growth of students, which is emphasized in many traditional course styles. The general attitude is that it does not matter who is completing the work as long as it gets done. This approach encourages students to set aside personal matters in favor of producing results, and it sets a serious professional tone for the design and execution of their ventures.

Students are expected to become experts on their ventures as soon as they choose, or are assigned to, a team. This role requires the students to both have the confidence necessary to accomplish all tasks as well as the humility to identify gaps in their knowledge and invite in outside experts. Teams spend countless hours, late nights, and weekends pushing their ventures 
and research projects forward. Throughout the beginning stages, students often become attached to their venture: many students described their venture as their "baby." This connection is essential for most students who need the extra motivation of feeling it is their duty to carry through the project that they see as having so much potential. Several students felt they "wanted to see the venture through and make sure it gets done" because they saw immense potential for their venture to have real impact in the field. Almost every interviewee expressed positive sentiment to their venture, with "it was a pretty important project" and "we were proud of it" being common responses.

Entrepreneurship is inherently chaotic and there are few step-by-step standardized procedures. Risk management and pivoting compound ambiguity and chaos in entrepreneurial settings. This setting favors individuals who are able to quickly adapt to a changing climate. Though feeling responsibility for their venture is beneficial, students can also become too attached to a specific business model, design, or presentation. This idea fixation can create tension within teams and hinders progress of the venture when that idea is no longer the best option. A team trying to sell nutritious pizza in Kenya exemplified idea fixation when members struggled to adapt their business model to the changes in daily operations. The inability to change the team's exit strategy from building a single business into franchising or selling the intellectual property created conflict between team members and led to inefficient use of time. This resulted in the ultimate failure of the venture. Another example of idea fixation arose for a team designing a data collection device. One team member often became too attached to his plans, and was unwilling to make design changes that others saw necessary. Conflicts caused by idea fixation slow the progress of a venture and lead to unnecessary tension. HESE teams are expected to document and defend design decisions, forcing them to understand the rationale behind approaches and pivot when the circumstances surrounding a decision have evolved.

HESE classes always include individual debriefing sessions with each team, including the professor. These sessions are used to update the professor on the team's progress, provide advice and direction for struggling teams, and discuss new ideas and proposals. By sitting down together as equals, everyone can share what they have accomplished, discuss what they are struggling with, and together formulate the next steps to be taken. The professor can also offer honest feedback to the teams. Sometimes this feedback can be critical and require teams to redo an entire deliverable at the last minute. Students often find this to be frustrating, as they believe that major issues should have been addressed earlier, and blame poor communication with the professor. Many teams push for more frequent communication through email and extra meetings, which also helps expedite the project in general. Within teams, regular meetings outside of class help ensure everyone is on the same page and making progress. Conflicts are often managed and avoided through clear communication among group members. Many students noted that a key to success when working in their teams was to allow every member to express their ideas, even if not all ideas were acted upon. 


\section{Team Building}

Over the years, the HESE program has refined the process of retaining veteran students while integrating new ones each semester. This method is used in the hope that each student will "fall in love" with their venture and enjoy the research while being motivated and mentored to work on something they think really matters. This process does not always have the intended result because some teams do not work well together or have students who are not as interested or invested in the venture. There is room for improvement in the team creation process to incorporate people's personalities into team determination to minimize intra-team conflicts later in the semester.

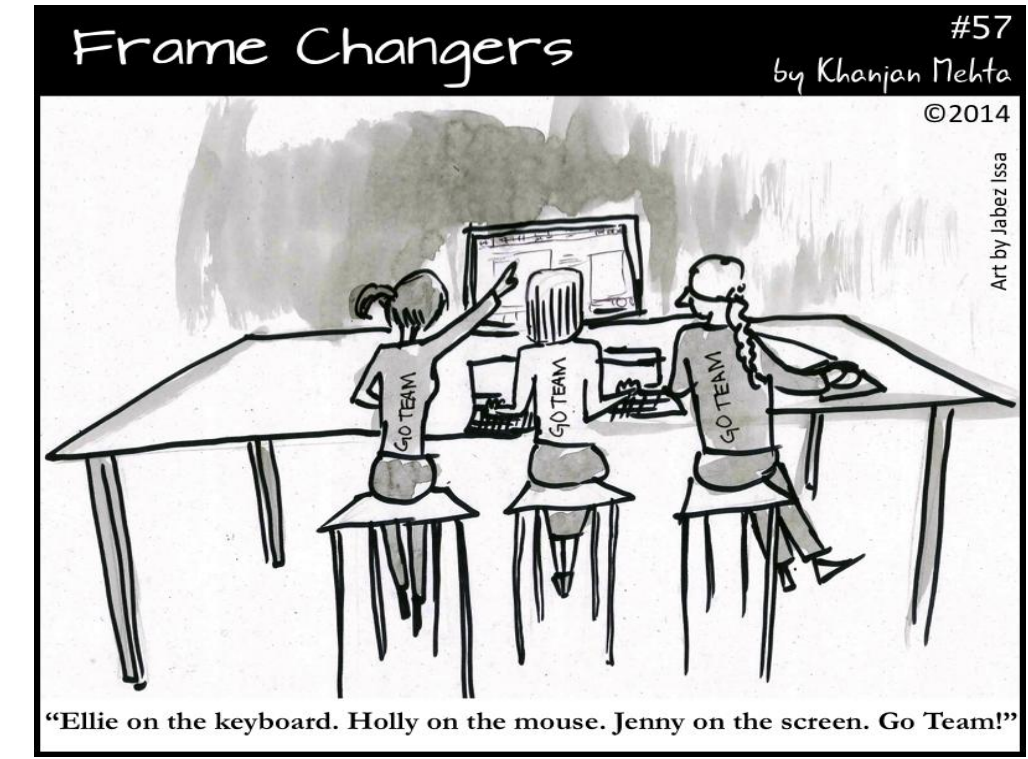

FIGURE 3

STUDENTS CANNOT ALL WORK ON THE SAME TASK. RATHER THEY WORK ON DIFFERENT TASKS TOWARDS ONE COMMON GOAL.

The team building experience in this course is like none other for many students who have only experienced teamwork in the form of short-term, temporary assignments in a general education or major-specific course. The multi-disciplinary nature of HESE teams directly contrasts to student experiences in traditional courses where they are paired with others within their own major. In HESE, it is not uncommon to have engineering undergraduates working alongside medical students or those seeking degrees in agricultural sciences or international affairs. These student teams stick together for at least one 15-week semester and up to 1.5 years, even longer if they stay involved for multiple years or become HESE Fellows after graduation. They often live, eat, work, and travel together during fieldwork. Teambuilding is thus essential from the very beginning in order to work harmoniously and make continuous progress. Each team develops a form of regular communication. This can be by group text, an email chain, and/or regular meeting times. Consistent and continuous communication allows everyone to catch each other up on the work they have been doing, share the problems they might have, and remain engaged while ensuring the work is shared openly. Candid communication helps prevent frustration in students who feel like their ideas aren't being heard or their input isn't valued. All current teams share an internal file which contains the email address and phone numbers of all 
members, including professors. This ensures consistent, open contact and prevents individuals from avoiding their team. All communications between the team and the faculty member are shared with all members, via group emails, to avoid any miscommunication.

The tasks and obstacles faced by the teams are so varied that students are quick to split up work based on the strengths of individual team members. "We let people gravitate to what they're good at," explained one student. Sometimes this division of labor is based solely on major, but more often it is determined by student interests and passions, which do not always align perfectly with their academic major. Most students find themselves being tasked in at least one thing that they have never studied. For example, a biology student taking the lead on the business model for the venture or a liberal arts student taking over the reins of developing the clinical testing strategy for a biomedical device. Students usually self-identify their interests, and over time, teammates learn to trust and respect each other's strengths. Groups find this strategy convenient when the strengths of some members cancel out the weaknesses of others, but in some instances, says one student, "everyone felt outside of their comfort zone, none of us had experience in what we were tasked with." In these situations, strengths can become harder to identify and morale harder to maintain. In the end though, most teams learn more about crossfunctional and interdependent work on these teams than on their major-based homogenous teams.

Team roles are also adapted as ventures progress and students discover which approaches work and which do not. Group leaders emerge organically based on the passions and personalities of individuals, not necessarily on age or experience. For example, an aerospace engineer may find himself as a leader of an agriculturally-focused venture due to his own drive and interest in its success. Teams create their own short-term objectives and set their own deadlines. This level of independence forces teammates to be accountable to each other first, and then to external stakeholders.

While teams faced obstacles and problems that are usually beyond the scope of anything they have faced in other classes, having a common adversity often helps maintain morale and motivation. At the beginning of a semester, teams often bond over one common adversity: the professor. When challenged immediately with high expectations, students find solace in sharing the burden amongst the group, and band together to meet the requests or decide to defend their own views. For example, the day prior to an important design presentation the professor advised one team to almost completely overhaul their slide order and design. In order to adapt their presentation, the team banded together and spent the next several hours methodically working into the night to address each recommended change for the presentation the following day. This enormous effort was based completely on trust in the professor's judgment because ultimately, an external referee panel was going to grade them and the professor had no say in it whatsoever. As the semester progresses, students realize how this flipped classroom setting works and how the professor, advisors and even the referees are all collaborators helping the team get their innovation to the marketplace.

At other times, particularly later in the semester, teams are more willing and able to scrutinize opposing expectations and mount defenses for their own ideas. One group of students successfully convinced the professor to add a community-based electronic payments system as a project in the Social Entrepreneurship class. They defended their venture successfully to all the referees and developed a truly innovative system that has already received ground validation and has a pending proposal with the Gates Foundation. Another common adversity is the presence of an unengaged team member who does not do his or her share of the work. This obstacle can 
bring the other team members together as they attempt to make up the lost work or find ways to deal with the problematic student. Faculty mentors get engaged in such situations and try to find equitable work distributions. More often than not, consistently low-performing students end up with low-priority tasks and get sidelined. Sometimes they find their motivation again and reengage with their team while at other times, they drop the class and pull out of the program.

Creating strong, trusting relationships influences how team members communicate and work together. During the fieldwork experience, dinner each night is prepared through team effort. Contributing to the preparation and observing the teamwork involved in each meal reinforces a strong and healthy team dynamic. Team members also find ways to bond over their similarities. Students who join the HESE program usually have some similar motivations, goals, or attitudes that lead them all to this program. For example, one-third of the students tend to pursue medical school, another third intend to pursue graduate school while the final third pursue law school, entrepreneurial opportunities or regular industry jobs. An increasing number of students pursue Fulbright Fellowships, Peace Corps, Teach for America, and similar programs. Uncovering what they have in common is a great way to deepen relationships and usually occurs as a natural consequence of the sheer amount of time students spend together.

Common disruptions within groups are personality clashes, unmotivated members, and a sense of entitlement. Personality clashes may or may not be avoidable by increasing group communication and developing friendships. Students invest so much time into these ventures that emotions will inevitably run high when dealing with frustrations and changing plans. Sometimes, an individual will take out their frustrations on other team members. A common manifestation of this is a student fixating on an idea and thus struggling to pivot quickly. Idea fixation is usually a result of students failing to separate professional and personal motivations, which often leads students to feeling hurt or offended when the team decides to go in a different direction. Another personal issue students encounter are differences in working styles. For example, one student may be well accustomed to working late into the night and will expect a teammate to do the same. If the teammate does not share this work ethic, he or she may be perceived as a slacker. Some students struggle to embrace the highly iterative nature of the design process. They want to do a certain task once and be done with it. These differences should be acknowledged and embraced by employing different working styles for different tasks, instead of allowing them to create conflict. This is often easier in smaller groups than larger ones. Larger groups also run the risk of forming cliques or creating the appearance of "ganging up" on someone.

Successful teams learn how to attend to, and manage emotions, during stressful times. Teams accomplish this best when students become friends. Teammates encourage each other when things become difficult, which ultimately helps them achieve their end goals because it motivates every individual to pull their own weight. They know when to work hard, but can have fun together when they need to relax. In addition, individual students are encouraged to take a break when necessary to manage their stress level. This separation of professional and personal motivations allows for teammates to act both as reliable coworkers and personal support groups. This dual role allows them to address each other's frustrations and troubles without creating serious conflict. It also helps team members deal with critical feedback from team members, professors and judges. This is often a steep and painful learning curve for students who have not experienced scathing, and often conflicting, professional criticism and downright failure. The ability to take feedback professionally and not as a personal attack is a key skill for students to develop as it enables them to effectively adapt and grow. 
Typically, $20 \%$ of the students drop HESE classes during the first week once they realize what the expectations are. However, a few students who are wholly unmotivated and unfit for this level of engagement and teamwork still hang on. This dynamic pushes more motivated individuals to take on the leadership roles and work with their team members to delegate work appropriately. Issues with students not completing their work detracts from team progress and can create disruptions that distract from their main goal. Sometimes groups will address that member directly, with mixed results. Since the focus of the program is on real-world outcomes and not grades, teams will often stop assigning work to unmotivated workers in order to continue making progress. These students may end up dropping the class entirely, and work will be redistributed among the remaining participating team members. "One team member left halfway through semester," recalled a recent graduate. "He was struggling for a while with the workload. We tried to help by slowly reducing his workload, but we had to eventually cut him out. It wasn't even worth having him on the team."

Having teams composed of both graduate students and undergraduate students can lead to an unwanted hierarchy within the team. In some cases, graduate students and NSF research fellows refused to do what they perceived as "menial tasks like building the prototype in the machine shop" and reassigned that work to undergraduates. This sense of entitlement amongst graduate students hinders the progress of the venture by ignoring the valuable perspectives of the undergraduate students, delaying the completion of basic tasks, and causing intragroup tensions to rise. Making expectations clear from the beginning for the team and each individual helps to alleviate some of these kinds of issues.

One of the major challenges in having multi-year ventures is the high student turnover rate. Having an almost entirely new set of students involved in the program each year means that information is at risk of being lost in the transition. To avoid this, steps are taken to minimize tacit and maximize explicit information. The primary way safeguard information is to encourage research and publications. By encapsulating a team's progress in a concise and precise manuscript, future students are able to easily learn from previous work and do not have to waste time searching for information or repeating research. Publishing also allows a wider audience to benefit from the insights gained by each specific team. In addition, there is some continuity in students who return to HESE as veteran students, teaching interns, Fellows, advisers, and collaborators. They are able to use their previous experiences to guide incoming HESE students and facilitate the transfer of knowledge from semester to semester.

\section{CONCLUSION}

The primary motivation for the development of the HESE program was to engage students in the design of socially-relevant technologies and launch sustainable and scalable enterprises that address global developmental challenges. A unique academic structure and nontraditional faculty roles help create an intense, impact-driven culture that consistently and successfully nurtures multidisciplinary teamwork. While teams still face common obstacles such as personality clashes, unmotivated team members, and chaotic environments, open communication and the desire to create real-world impact gives teams the motivation to overcome these issues in favor of achieving ambitious outcomes. Students consistently respond that they would recommend the program to other students, though often with the caveat that they be prepared to work hard and have an open mind. Further development of the team selection process and professor-student communication will decrease team conflicts and frustrations. Overall, the successes in bringing 
together high-performing multidisciplinary teams can be attributed to the singular, almost obsessive and arguably delusional, focus on real-world social impact.

\section{ACKNOWLEDGMENTS}

The authors would like to thank Kate Holmes, Ellie Kriner, Greg Wenner, Boyd WarwickClarck, Martin Marino, Kaylyn Hannon, Phillip Sundin, Steve Suffian, Rachel Dzombak, Siri Maley, Mena Abdelmalak, Bilaal Ahmed, Grace Warkulwiz, Josh Bram, Selva Jeganathan, Will Dawson, Richard McLaughlin, Sarina Katz, Eric Obeysekare, Staci Sutermaster, and Nick Natoli for sharing their teamwork experiences and lessons learned with our team. Siri Maley, Steve Suffian, and Rachel Dzombak provided constructive feedback on the manuscript. 